\title{
RESEARCH OF THE PRESENT AND EMERGING APPLICATIONS OF SMART ROBOTS AND UNMANNED AERIAL VEHICLES IN THE AGRICULTURE DOMAIN
}

\author{
Nikolay Zlatov1, Georgi Hristov², Diyana Kinaneva2, Yavor Yotov ${ }^{1}$ and Plamen Zahariev² \\ ${ }^{1}$ Institute of Mechanics - BAS, Acad. G. Bontchev St., 1113 Sofia, Bulgaria, \\ ${ }^{2}$ University of Ruse, Studentska str. 8, 7004 Ruse, Bulgaria \\ Emails: zlatovn@hotmail.com ,javor.jotov@imbm.bas.bg \\ \{ghristov, dkyuchukova, pzahariev\}@uni-ruse.com
}

\begin{abstract}
The last fifty years humanity has witnessed significant progress in the development of smart and autonomous robotic systems. This advancement was partially driven by the numerous studies and innovations in the area of the computer systems, the development of new communication technologies and the evolution of the electromechanical systems. All of this has paved the road for the slow but steady acceptance of the robotic systems as replacement for the human labour, especially for time-consuming, exhaustive or hazardous activities. Nowadays, the robotic systems are used for many different activities in almost all industrial and manufacturing domains. The agricultural domain is among those where the robotic systems are used the most. In the present time, ground and areal robots are helping in many farming processes and are resulting in higher crops yield, better weed control, improved environmental monitoring, etc. This paper presents a review on the most notable present applications for smart robots and unmanned aerial vehicles in the agricultural domain and highlights several emerging solutions as well.
\end{abstract}

Keywords: Smart Robots, Unmanned Aerial Vehicles, Precision Agriculture.

\section{Introduction}

The evolution of the information and communication technologies (ICT) and the advancements in the electromechanical systems have led to a new industrial revolution. This made possible the slow integration of automation and robotic systems in different work processes and in the everyday human activities. The agricultural domain, as one of the most important for humanity, became also one of the most significantly impacted by these new technologies. In the last fifty years, numerous conceptual models, prototypes and mass-produced robots have been developed and used for all sorts of agricultural activities. This contributed for the birth of the precision agriculture. This collective term is used to describe the process of livestock and crops management using different modern-day ICT, automation and robotic systems.

At the present time, the precision agriculture is considered as an approach that overcomes the constraints of traditional farming and provides means for better time management and use of the agricultural machinery. Undeniably, the increase of the harvest volumes and the preservation of the environmental conditions are the main result from the global application of the precision agriculture technologies. However, there are also some research papers and surveys [1, 2], which suggest that the rate of adoption of the precision agriculture is relatively low, mainly due to the increased complexity of the end systems and their higher purchase and maintenance costs. Moreover, these studies present the adoption of the precision agriculture as solution for specific or dedicated issues and not as a complete and fully implementation-ready technology.

With the recent advances in the information and communication technologies, many of the developed solutions were taken and integrated in the agricultural machinery. The largest penetration of the ICT was made in terms of the vehicle guidance and autopilot systems. This is because there are numerous commercially developed and widely available hardware elements and software systems, which are adaptable for the agricultural sector and require less investments. Nevertheless, precision agriculture is very complex and multidisciplinary science that requires the involvement of agronomists, software developers, designers, telecommunication experts, mechanical and electronics engineers and many other. 
The remainder of the paper is organized as follows - the second Section of the paper presents some of the present applications and the challenges for the ground-based and the urban robotic systems, which are used in the precision agriculture domain. The third Section of the paper presents the use of the unmanned aerial vehicles (UAVs) for different agriculture activities. Some of the emerging applications for smart robots and UAVs in the agriculture domain are presented in the last Section of the paper. The paper is then concluded with discussions on the presented analyses and with recommendations for further work.

\section{Present State of the Agricultural Robot Systems}

\subsection{Ground-Based Robots for Open Space Agriculture Applications}

Precision agriculture is one of the domains that has taken full advantage of the modern ICT systems.
The agricultural robots became the recipient of the majority of the smart solutions, which were focused on better navigation algorithms, crop mapping, soil sampling and mechanical manipulations of the ground (Fig. 1).

Other notable applications are the crops harvesting actions and the collection of fruits and herbs, as well as the precision farming actions, which include all heavy workload operations, like tillage, seeding, planting, etc.

The main development efforts for the groundbased robots for precision agriculture are focused on the improvement of their autonomous movement and navigation capabilities. There are several different approaches for autonomous navigation, which are used by the majority of the robots in this category, but they are all based on the algorithms that were developed for military purposes [2]. One of these approaches implements satellite triangulation, which allows the robot to move through the open field towards the desired endpoint, as well as to follow specific routes or a set of waypoints.
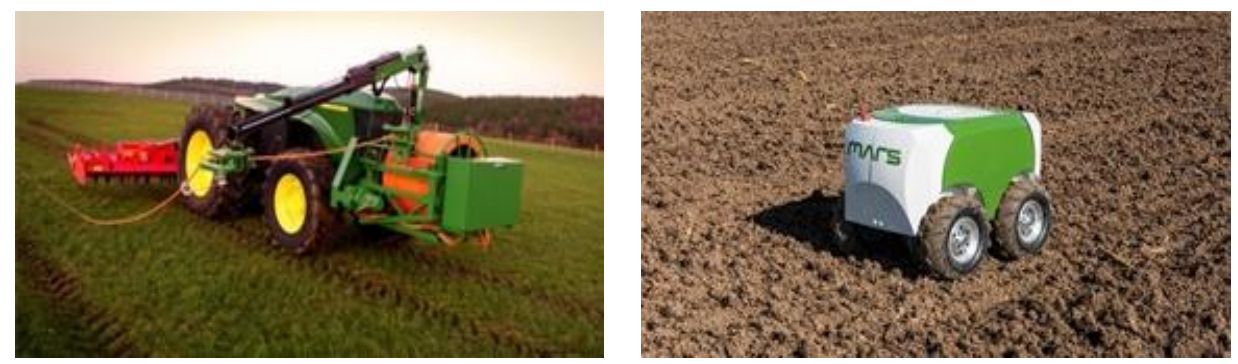

Fig. 1. Autonomous grid powered electric tractor by John Deere (left) [3] and the MARS robot while performing seeding operations (right) [4]

The satellite navigation approach is very simple to implement and is supported by many manufacturers of agricultural machinery. However, it has many disadvantages, especially when used on its own. Some of these disadvantages are the lack of updated terrain information, the unavailability to analyse the surrounding environment and the unavailability to avoid obstacles. For this reason, many of the modern agricultural machines, both piloted and autonomous, have additional visual feedback control, which is implemented using optical or electro-optical cameras [5], ultrasonic proximity sensors or even LIDAR (Light Imaging, Detection and Ranging) systems.

The improvement of the actuators of the field robots and more specifically the improvement of the precision of the tools used for seeding, transplanting, fertilizing and harvesting is another extensively studied and researched area. Many different manufacturers are focusing on the precision of their harvesting tools, which will ultimately increase the volumes of the harvested crops. Others are using cloud-based solutions and specialized sensors to determine the proper course of actions or the required level of fertilizers [6].

Many of the mentioned technological solutions are used on semi-autonomous agricultural systems and some of them even require constant human observation and/or management. This is due to the complexity of the systems, which require also the use of more advanced hardware and software solutions. The proper selection of the hardware of the end-user smart agricultural system has a strong impact on the robot performance and its complexity and contributes to the overall system costs. All ground-based agriculture robots can integrate ICT systems for GPS, odometry, line guidance, path planning and remote-control as solution for their navigation strategy. This is possible thanks to the modern auto-control and auto-pilot microcomputers, which were developed primary for remote control purposes.

However, the "brain" of the agricultural robot is not the only hardware concern. The movement of the robot is also a significant challenge. Crawler and caterpillar platforms are some of the feasible options 
to solve this issue, but the wheeled robots remain the most widely available solutions - due to their simplicity.

\subsection{Robots and Automation Systems for Greenhouse Agriculture}

Greenhouse farming is often a suitable field for applying the technologies of automation, computing and robotics. Some examples are the control of temperature and humidity, the soil preparation and the supply of water and nutrients. However, all of these are not purely robotic functions, but rather solutions for automation using microcomputers and specialized sensors.

The real greenhouse farming robots were developed to perform specific tasks, which are either hard or dangerous for the human workforce, due to the harsh conditions of the greenhouses. Some of these tasks include environmental monitoring and control, crop monitoring, supply and treatment of nutrients, pest and disease detection, etc.

The environmental monitoring of the greenhouses is important for the control of the growth of the crops and helps determine the traceability and the quality of the products. Nowadays, most of the systems that are used for environmental monitoring in the greenhouses rely on industrial or wireless sensor networks.

In the recent years, the indoor robots are becoming more and more popular, as the traditional human labour force is focusing on more highly paid intellectual and ICT related jobs and are trying to avoid the exhausting agricultural work. Commonly, the tasks of the robotic platforms are limited to the manipulation of just one fruit or vegetable, mainly due to the limitation of the technological solutions and the restricted working spaces.

The design of the greenhouse robots is also different from the design of open space agricultural machines. These robots are two types - mobile (Fig. 2) and fixed (Fig. 3).

They typically have one or more manipulators, which are under the form of extendible arms or are represented by a fixed head that moves in all three directions.
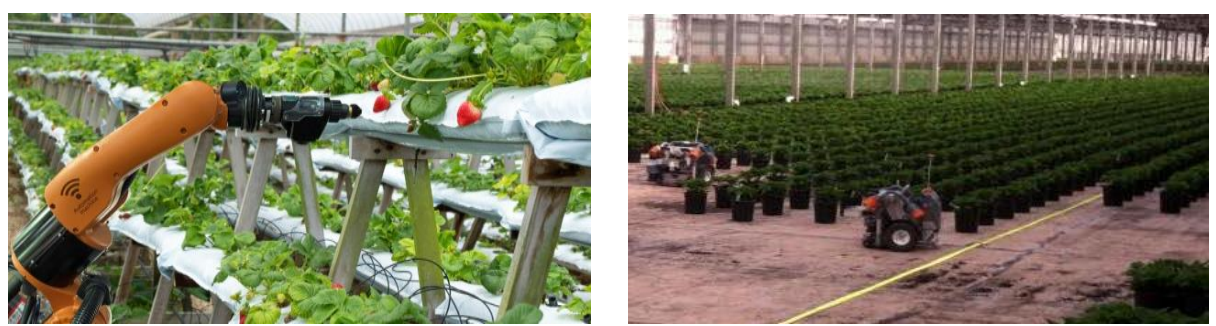

Fig. 2. Specialized greenhouse farming robot with manipulator arm (left) [7] and indoor greenhouse farming using mobile miniaturized agricultural robots (right) [10]
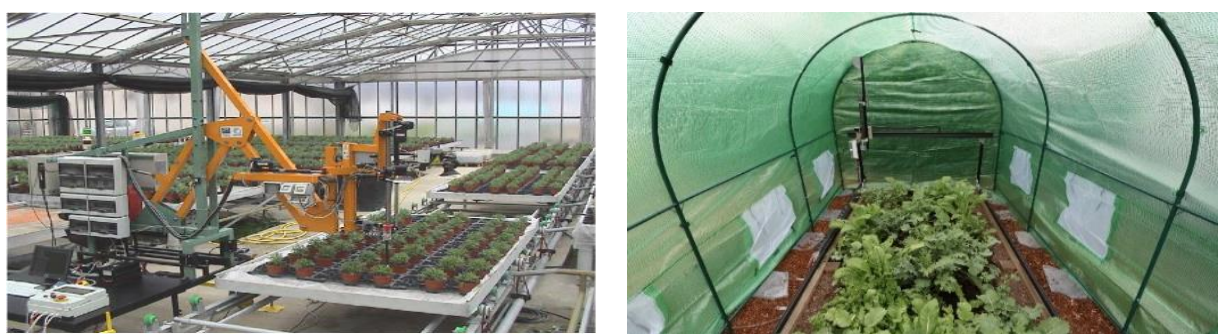

Fig. 3. Fixed position greenhouse farming robot with extendable arm and exchangeable manipulators (left) [9] and the FarmBot open-source smart gardening and farming robot (right) [8]

\subsection{Robots for smart Farming and Precision Agriculture in Urban Areas}

Urban farming is a collective term for the farming processes in dense urban areas. These processes can be achieved in the gardens of the houses, on the rooftops of large buildings or indoors in specialized facilities for stacked or vertical farming. Urban farming and vertical farming are two trends that are getting very popular in the modern societies. Vertical farming alone was valued at 1.782 billion USD in 017 and is expected to reach 10 billion USD by 2025 [8].
This trend presents the great potential of the urban farming market. For this reason, many manufacturers of agricultural systems and machinery have started to explore and analyse it in the last couple of years.

At the present time, there are two types of urban robots - static/fixed cell robots and mobile robots (Fig. 4). These platforms differ significantly in their design and functionality. Also, some of them are available as commercial products [11], while others are in their development stage or are available as scientific evaluation prototypes only [12]. 

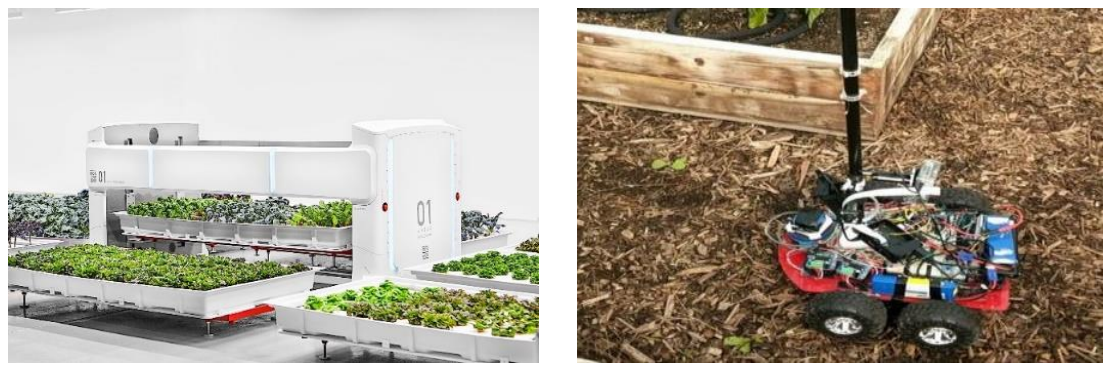

Fig. 4. The Iron Ox Angus Module mover for indoor or vertical farming (left) [11] and the prototype of the Farmaid plant disease detection robot (right) [12]

The mobile urban agriculture robots are becoming increasingly accepted and more available, as many of their hardware and software components are already commercially available and used in the construction of their bigger counterparts - the open space agricultural robots. The main challenges for these robots are the limitations of the physical environment and the need for improvement of the functionality of the robots. Many of the mobile urban agriculture robots are implementing different computer vision approaches, which allow them to analyse the surrounding environment or to perform visual examinations about the condition of the plants (Fig. 4).

The fixed or cell robots are a newly emerging trend and are characterized by their significant production volumes. The robots are also usually very large in size, which makes them suitable for halls and buildings (Fig. 4). These robots are designed to work with all sorts of crops, which are positioned underneath them. The fixed cell urban farming robots are capable of operating during the complete development cycle of the crops. Nevertheless, they are more expensive, have higher maintenance costs and are more difficult to support [10].

Urban and vertical farming are two very interesting agricultural areas, which will have vital role in the future years. They are also planned to be used in space and for planet colonization missions.

\section{Unmanned Aerial Vehicles for Precision Agriculture 3.1 UAVs for Environmental Monitoring and Animal Husbandry}

The precision technology has driven the farming revolution of the recent years. The capability to monitor the crops from the air added new possibilities to the agricultural domain by providing priceless information to the farmers and resulted in significant increase of the crop yields. With a single flight of an unmanned aerial vehicle (UAV), any farmer can receive dozens of highly accurate photographs of agricultural lands that cover hundreds of hectares and all of this without the difficulties related with the manned aerial services.
The obtained photographs usually have higher image resolution, compared to these provided by satellite or other areal services, and are not influenced by the cloud cover. Using image-processing software products, the aerial photographs can be combined into large orthomosaic images. With the help of specialized algorithms, like the Normalized Difference Vegetation Index (NDVI), a detailed reflectance map of the crops can be created. This map is the key for better yields, lower costs and pushes the agricultural business forwards. This technology is used to pinpoint the areas of land that need closer examination - meaning less time spent scouting the crops and more time invested in treating the plants that need it.

The results, obtained after the processing of the images taken from the UAVs, can be used to identify crop health and stress. The early detection of problematic areas within the crops means also that an early and local treatment can be applied. Plants need the correct levels of nutrients in order to thrive and produce a strong yield. The correct levels of nitrogen can ensure strong growth of vegetation and foliage, the correct levels of phosphorous are required for strong root and stem growth and the correct levels of potassium are needed to improve the resistance to diseases and to ensure the betterquality of the crops. If any of these nutrients are lacking in the soil, the plant will become stressed and will struggle to thrive. The NDVI index mosaics are offering the ability to identify exactly which areas of the crops are stressed or struggling, which means that the farmers can target and work on these areas. The multispectral imagery provided by the UAVs can identify these problematic areas long before the issues become visible to the naked eye. This means that the problematic zones can be targeted much earlier before the crop development and yield is affected.

Excess moisture has always troubled farmers. Field imaging for the purpose of drainage ditching and water control has provided a mean to solve this issue. The solution requires the use of an UAV, which is used for the creation of a RGB orthomosaic of the problematic farming area. This image is usually reconstructed from separate high-resolution photographs, which are taken at altitudes between 
50 and $100 \mathrm{~m}$. The images are processed using specialized software and are used to create visual and geo-referenced maps of the field, as well as a Digital Surface Model/Map (DSM), which represents the triangulated elevation of the field. Based on these maps, various calculations and algorithms can be applied to provide elevation maps, hill shade renders and watershed analyses, which can be used for identification of potential areas that will be impacted by excess moisture and need attention to ensure better drainage. In this way, adequate measures can be taken and the crops will not be significantly affected.
Advances in the aerial equipment and technology have made the UAVs a key part of conservation strategies for marine reserves, forests, deserts and many other landscapes.

Drones are also widely used for remote observations of the habitats and the daily lifecycle of many endangered species. All of this is possible with the help of different sensor technologies and in combination with variety of cameras.

The most widely used types of cameras and their typical application areas are given in the table below.

Table 1. UAV camera types used for monitoring of wildlife and ecosystems

\begin{tabular}{ll}
\hline Camera type & Main application \\
\hline High resolution, low distortion & Aerial mapping and imaging, photogrammetry and 3D \\
RGB camera for images and video & reconstruction, plant counting, surveillance, surveying \\
Multispectral camera with & Plant health measurement, water quality assessment, \\
narrowband filters for Blue, Green, & vegetation index calculation, plant counting \\
Red, Red Edge, Near-Infrared & Heat signature detection, livestock detection, surveillance and \\
& security, water temperature detection and water source \\
Thermal & identification surface \\
& 3D digital surface modelling, stockpile calculation, surfaring \\
& variation detection and flood mapping \\
LiDAR & Plant health measurement, Water quality assessment, \\
& Vegetation index calculation, Full spectral sensing, Spectral \\
& research and development, Mineral and surface composition \\
Hyperspectral camera & surveys
\end{tabular}

Preservation of the flora and the fauna has always been an important process, especially if it is involving endangered species. NIR and the NDVI images from UAVs can be used for monitoring of the vegetation in a given region. Based on these images, the necessary means for preserving specific sorts of plants, bushes or trees could be applied much easier and much faster. The NDVI images also could be used for localization of invasive species, which would allow for a much earlier response to their spreading.

UAVs could also play important role for observation or monitoring of wildlife or domestic animals. Thanks to the modern technologies, the UAVs are almost noiseless, compared to airplanes and helicopters and cause less stress to the animals.

\subsection{UAV-based Solutions for Seeding and Spraying of Crops}

In most cases, the agricultural UAVs are used for environmental monitoring and assessment of the health and the condition of the crops. Nevertheless, there are also several examples of applications where the drones are being actively involved in the agricultural processes. One such example is the Agronator drone [13], which is capable of carrying payloads for seeding and plant nutrition (Fig. 5). Another great example is the DJI AGRAS T20 [14] unmanned aerial vehicle (Fig. 5), which is capable of caring up to 20 litres of liquids and can conduct autonomous operations over a variety of terrains.
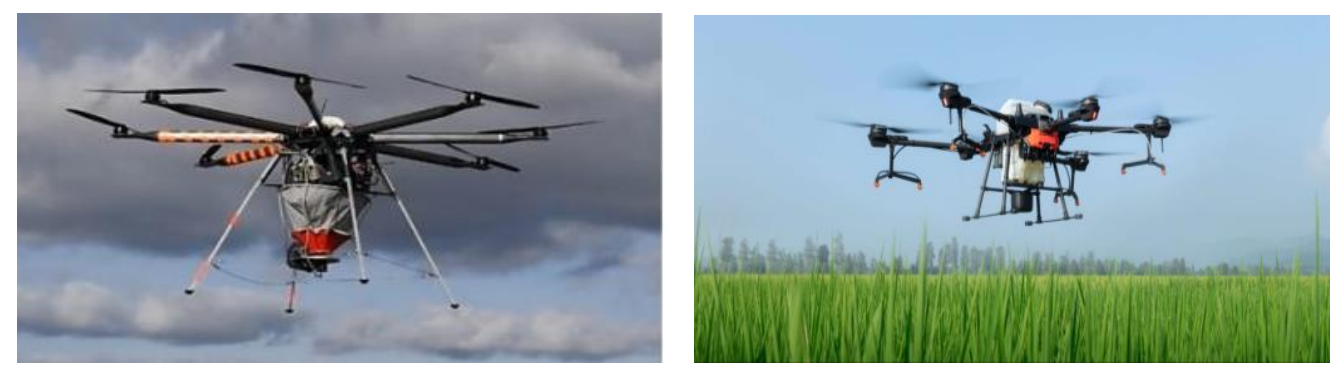

Fig. 5. The Agronator unmanned aerial vehicle (left) [13] and the DJI AGRAS T20 (right) [14], which are used for seeding and for spraying of pesticides and fertilizers to the crops 
According to a study made by the Food and Agriculture Organization of the United Nations and the International Telecommunication Unit [15], the use of the unmanned aerial vehicles for seeding and planting activities brings down significantly the costs for these activities. The drones can also be used to shoot seeds with nutrients in the soil with an average uptake of 75 percent, which is also discussed in the same study [15]. This further increases the efficiency of the planting process.

The drones that are used for planting, seeding and spraying, like the AGRAS T20 [14], are equipped with omnidirectional radars and are able to detect obstacles from all horizontal directions. In this way they can automatically circumvent objects, like trees, poles, etc. in their flightpath. With the help of LiDAR systems, the drones can monitor and follow the underlying terrain, which provides much higher level of autonomy.

\section{Emerging Applications for Robots in Agriculture}

The sections above have presented some of the most interesting present applications of robots and unmanned aerial vehicles in the agriculture domain. Nevertheless, the ongoing studies and the rapid advances in the ICT sciences are constantly leading
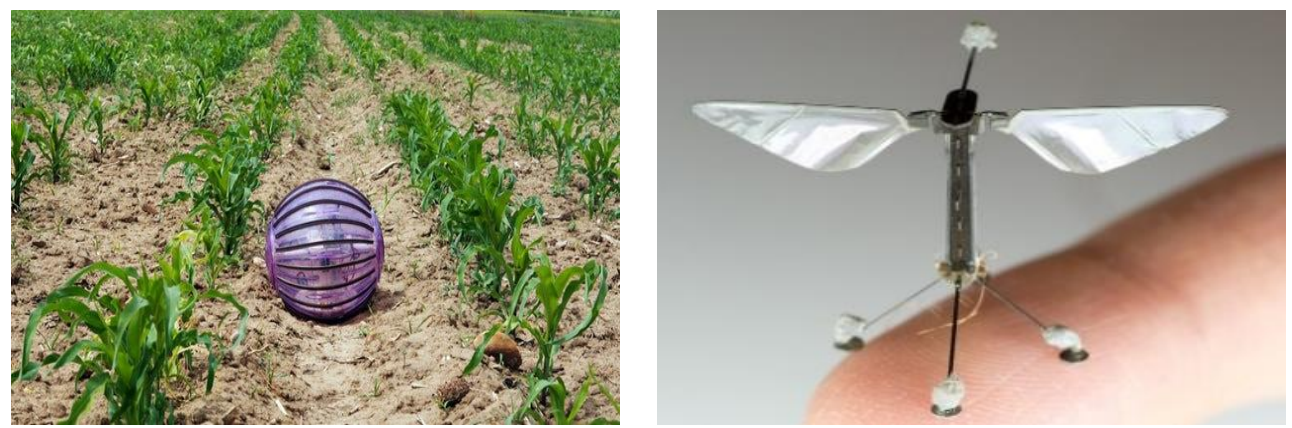

Fig. 6. The ROSPHERE robot (left) [16] and the Harvard RoboBee (right) [18]

Other interesting agricultural robots are designed using bioinspiration, which tries to replicate the biological evolution of animals or insects. For instance, hexapod insects are able to walk on all terrains, which can be used for the development of advanced physiognomies and walking patterns.

This is the case of Prospero, which is a prototype of hexapod robot that can plant, tend and harvest autonomously [17]. Another example is the RoboBee of the Harvard University (Fig. 6), which is micro-aerial robot inspired by bees [18]. Multiple such robots can be used for distributed environmental monitoring and assistance in crop pollination processes. to new possibilities and additional applications for these systems are emerging constantly. This section of the paper aims to present some of the emerging applications for drones and robots in the agriculture domain.

\subsection{Specialized Robots for Agricultural Purposes}

The traditional ground robots present several limitations, which are related to the constraints of the wheeled and the caterpillar motion paradigms. Additionally, the use of aerial robots is not always possible, especially when the task should be performed from a greater distance. However, there are several studies, which present agricultural robots with alternative locomotion systems [16, 17 and 18]. Sphere robots are driven by instability.

They are suitable and used in several applications and scenarios, including exploration and surveillance. The ROSPHERE robot (Fig. 6) is one such robot that is described by its authors as a new low-cost spherical robot for measuring soil temperature and moisture in precision agriculture [16]. In comparison to wheeled robots with similar size and capabilities, this robot is much lighter and robust in irregular terrains.

\subsection{Cooperative Robotics and Multi-robot Systems for Agriculture}

In numerous occasions, single robots are not able to perform complex tasks. Such examples are the activities that are time restricted or the activities that require coordinated actions in multiple locations [20]. In these cases, robot groups or swarms might provide some advantages over single robots or man-operated vehicles. This is especially important when effectiveness, efficiency, flexibility and fault tolerance is required.

Most of the cases, where multiple robots have to preform agricultural activities, are for homogeneous 
tasks, like mapping, ploughing, spraying of water or fertilizers, etc.

To use a fleet of UAVs instead of a single drone not a rare event and there are several techniques for area distribution and path planning, which can be applied. For instance, a team of small UAVs with low-cost cameras can cooperatively observe and localize domestic farm animals with the same accuracy and precision, as in the case where a single more advanced and better-equipped drone is used.

In some cases, the agricultural applications require heterogeneous robot groups. The most common situation is when the task consists of operations that are more effective from the air and other that are more effective from the ground (Fig. 7). For instance, aerial robots are able to cover efficiently large fields taking pictures and collecting data for the distribution of water or location of weeds, whereas ground robots can actuate on the crops with more robustness and precision and provide watering or applying treatments [19]. In another example, the UAVs can help in the localization of stranded animals, while ground-based robots can assist in the recovery and the return of the animals to their owners.

As evident from the examples, the heterogeneous multi-robot systems often combine the advantages and compensate the drawbacks of the different robots [20].

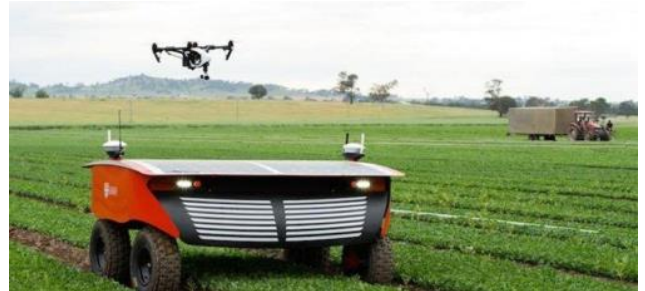

Fig. 7. A multirotor UAV is supervising a ground-based robot during field activities [19]

\subsection{Autonomous Inexpensive Small-scale Robots for Farming Activities}

The focus of the leading agricultural machinery manufacturing companies is the production of large and highly effective, but also very expensive autonomous solutions. For many small and mediumsized farms it is often very hard to buy these expensive robots. This opens a market gap, which can be filled by small-scale inexpensive robots. One such example is the AgRUbot prototype (Fig. 8), developed by the University of Ruse [21]. This solution is constructed using 3D printed parts and utilizes open-source computer vision and artificial intelligence methods for crop detection, analysis and classification. Furthermore, the robot has an onboard sprinkler system, which can be used for local treatment and precise delivery of nutrients or pesticides to the crops. AgRUbot is based on popular microcomputer systems, which makes it easy to program and manage.

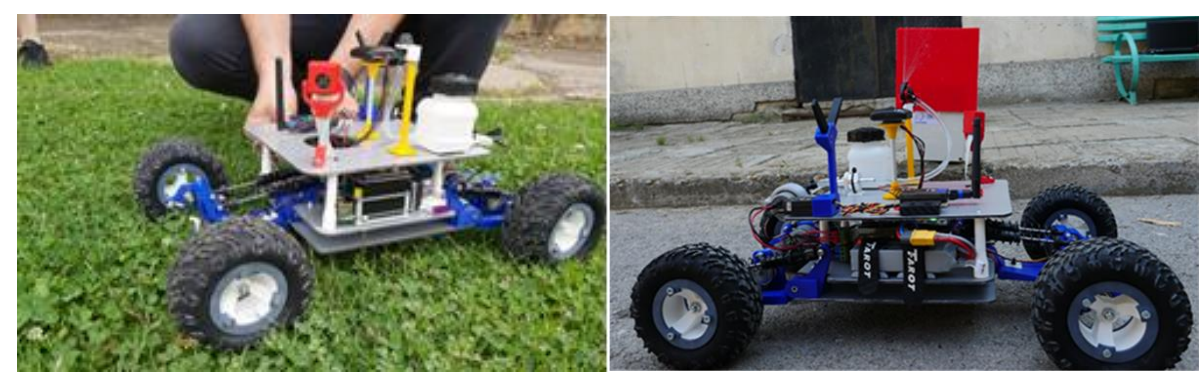

Fig. 8. The AgRUbot prototype during field tests (left) and while sprinkling liquid solution on a target surface (right) [21]

\subsection{UAVs for Forestry Applications and for Early Detection of Wildfires}

In forestry and nature conservation, the UAVs may be used for many applications, such as for detection of illegal tree harvest sites, for monitoring for trespassers, inspection of different forestry operations, monitoring of hard-to-reach natural forests, calculation of the tree biomass, observation of the vegetation growth and quality, control of invasive species, detection of river erosion, deforestation, landslide risk assessment, etc.

There are also several widely known methods for non-destructive detection of stress in the trees - by using airborne chemical and bio-sampling, by visual analysis, by fluorescence measurements, by optical reflectance measurements and by thermal infrared evaluations and all of these methods can be implemented using UAVs. Nevertheless, many of these applications require different flight follow-up processes, including image processing, development of georeferenced and orthorectified maps, etc. This makes the use of UAVs for forestry applications a difficult process, which is to be performed by experienced and trained personal.

In the recent years, another interesting application for UAVs in the forestry domain is becoming increasingly popular - the detection of 
forest fires and the post-fire assessment of the damage using drones. One such solution is the THEASIS system, which was developed under the "Forest Monitoring System for Early Fire Detection and Assessment in the Balkan-Med Area" (SFEDA) project [22]. The most extensive configuration of this system involves the use of two UAVs - a mediumaltitude long-endurance fixed-wing vertical take-off and landing (VTOL) drone that is constantly patrolling over the observed forest area and a secondary rotary-wing UAV for close inspection and confirmation of the detected fire.

This configuration of the THEASIS system was deployed and tested in the Rusenski Lom Nature Park in Bulgaria by a scientific team from the University of Ruse.

The general conceptual model of the system is presented in Fig. 9, while the used fixed-wing VTOL UAV and graphical user interface (GUI) of the system are presented in Fig. 10.

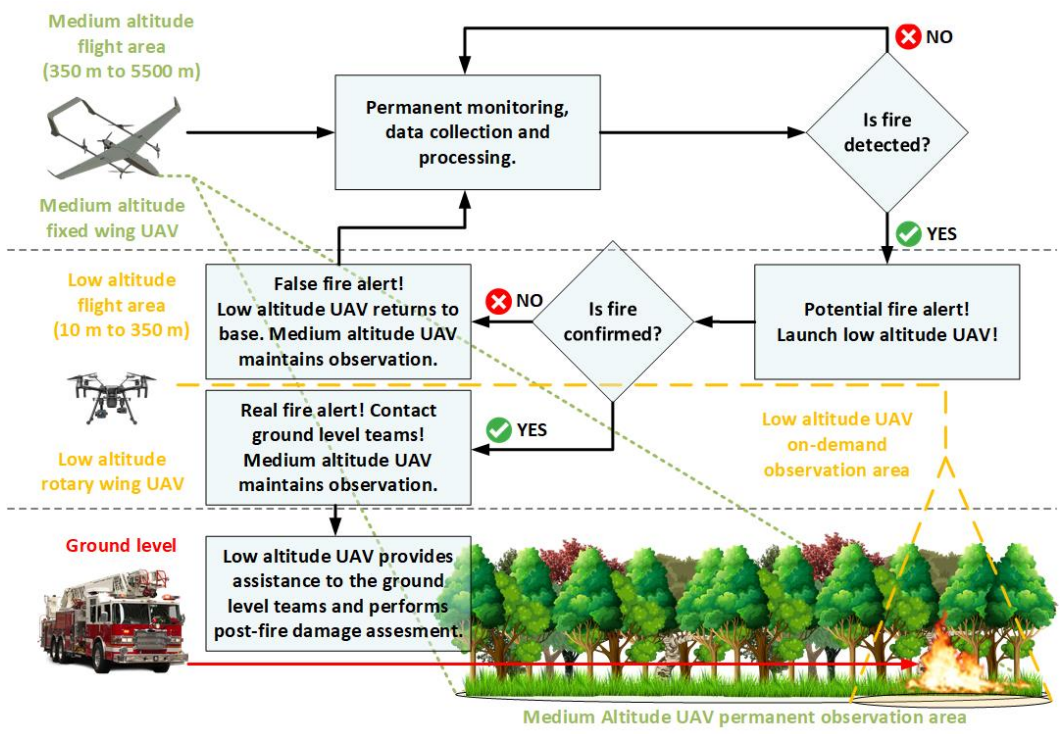

Fig. 9. The conceptual model of the THEASIS early forest fire detection system, which is based on fixed and rotary wing UAVS
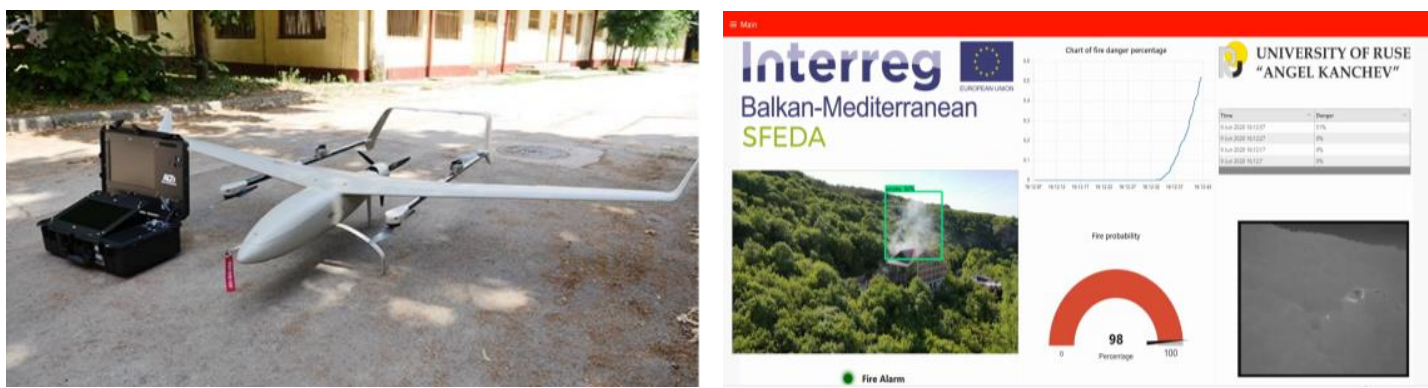

Fig. 10. The ALTi Transition F fixed-wing UAV [23] that is constantly patrolling the forest area and acts as primary source for detection of the potential forest fires and the GUI of the system that shows the output from the optical and the IR cameras, as well as the detection certainty

\subsection{A Novel Drone-based System for Fruit Manipulation and Harvesting}

The harvesting process is one of the most timeconsuming operations in the agricultural domain. While there are many robotic solutions for openspace harvesting, there are just few that are focused on the fruit manipulation and the harvesting of fruits from trees.

The majority of these harvesting systems involve the physical interaction with the tree, which includes the firm grip of the tree trunk and its hard or even violent shaking. In this way, the fruits can fall in collection bins or nets. While the process lasts only for couple of minutes, it can cause significant stress to the tree and can lead to broken branches, damaged tree bark and even in some cases to the breaking of the tree trunk. This all calls for a less invasive method that is both efficient and less harmful for the trees.

To answer the need for a solution to the abovementioned problem, a team from the Institute of Mechanics at the Bulgarian Academy of Sciences [24] is developing the DFTS [25], which is short for Dexterous Flight Telerobotic System. This solution is very interesting, as it involves an air-handling 
platform with a manipulator (gripper), which is lowered from an unmanned aerial vehicle and can perform local high-precision operations (Fig. 11).
The air-handling platform is gyrostabilized and can operate in windy conditions, which additionally increases the reliability of the system.
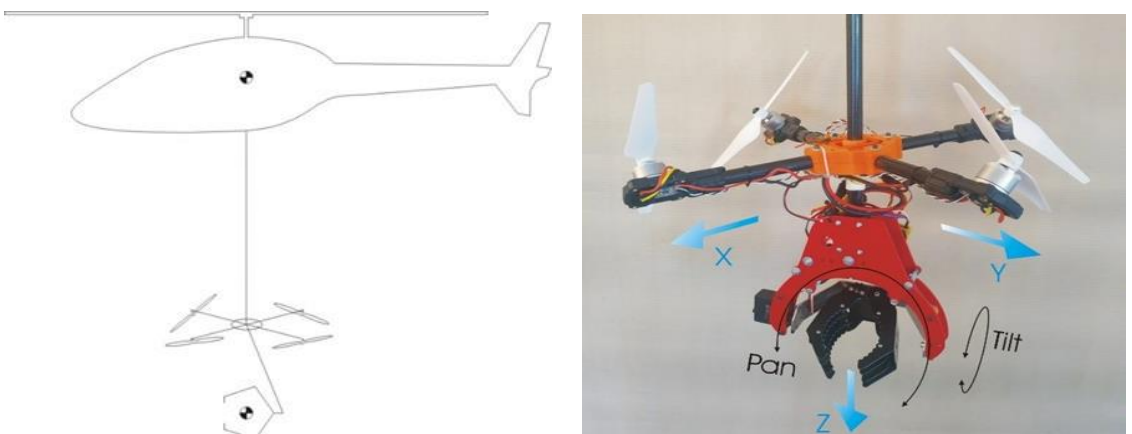

Fig. 11. The conceptual model of the DFTS (left) and the air-handling platform (right) [25]

The presented DFTS is flexible and can be used for collection of fruits, like coconuts, bananas, etc., which are growing in the crown of the trees. In this way, the necessity for a human to climb the tree is avoided and the process excludes any stressful interactions with the tree. Another possible application of the DFTS involves the replacement of the gripper with a spraying system, which is possible, as the air-handling platform is constructed in a modular basis. In this way, the drone can be used for local treatment of plants against invasive species of insects or vegetations, for assistance in the tree pollination processes, etc.

\section{Conclusions}

This paper presented an analysis on the modern robotic systems used in the precision agriculture. Some of the advantages and disadvantages of the ground-based robots, the smart urban robots and the unmanned aerial vehicles were discussed with connection to their use for different agriculture activities and in different farming processes.

The robot systems are subject to constant improvements, which leads to the development of new technologies for the power supply, navigation and propulsion of these human labour replacements. This made the agricultural robots more resilient, more flexible and very reliable. Regardless of this, the high exploitation and maintenance expenditures, the need of additional staff training and the restricted operation capabilities of the robots are preventing them from becoming a complete human replacement.

The wide acceptance of the artificial intelligence solutions has led to the integration of many new systems in the agriculture robots. With their help, these automation solutions are slowly progressing in new application areas. With the ever-increasing attempts of humanity to reach for the stars, the use of robotic systems is becoming imperative and it is just a matter of time before we see a robot operated selfsustained solution for off world agriculture.

\section{Acknowledgment}

This publication is supported by the Bulgarian Ministry of Education and Science under the National Research Programme "Smart crop production", approved by Decision of the Ministry Council №866/26.11.2020.

\section{References}

[1] Pierpaoli E, Carli G, Pignatti E, Canavari M. Drivers of precision agriculture technologies adoption: A literature review. Procedia Technology, 2013 vol. 8, pp. 61-69

[2] McBratney A, Whelan B, Ancev T, Bouma J. Future directions of precision agriculture. Precision Agriculture, 2005, vol. 6(1), pp. 7-23

[3] Official website of John Deere, https://www.deere.com/en/agriculture, last accessed 2021/05/07

[4] Official website of FENDT Mars robot, https://www.fendt.com/int/fendt-mars, last accessed 2021/05/07

[5] Vázquez-Arellano M, Griepentrog HW, Reiser D, Paraforos DS. 3-D Imaging Systems for Agricultural Applications - A Review. Sensors. 2016; 16(5):618. https://doi.org/10.3390/s16050618

[6] Weiss U., Biber, P., Plant detection and mapping for agricultural robots using a 3D LIDAR sensor, Robot. Auton. Syst. Vol. 59, 2011, pp. 265-273

[7] Emma Cosgrove, The State of Play for Farm Robotics, image recovered from the article published at the Agfundernews site https://agfundernews.com/farm-roboticsstartups.html, last accessed 2021/05/07

[8] Vertical Farming Market Overview, online article by Akshay Jadhav, 
https://www.alliedmarketresearch.com/vertical -farming-market, last accessed 2021/05/07

[9] Belforte G., Deboli R., Gay P., Piccarolo P., Ricauda Aimonino D. (2006). Robot Design and Testing for Greenhouse Applications. BioSystems Engineering, 95(3), 309-321

[10] Webpage of the FarmBot agricultural cell robot, https://farm.bot, last accessed 2021/05/07

[11] Website of the IronOx company, http://ironox.com, last accessed 2021/05/07

[12] Website of Farmaid - the Plant Disease Detection Robot, https://www.hackster.io/teamato/farmaidplant-disease-detection-robot-55eeb1, last accessed 2021/05/07

[13] Website of Agronator, https://agronator.de/en/, last accessed 2021/05/07

[14] Website of the DJI AGRAS T20, https://www.dji.com/bg/t20, last accessed 2021/05/07

[15] e-Agriculture in action: Drones in agriculture, edited by Gerard Sylvester, published by the Food and Agriculture Organization of the United Nations and the International Telecommunication Union, Bangkok, 2018, ISBN 978-92-5-130246-0s, http://handle.itu.int/11.1002/pub/8111728een, last accessed 2021/05/07

[16] J. D. Hernández, J. Barrientos, J. del Cerro, A. Barrientos, and D. Sanz, "Moisture measurement in crops using spherical robots," Industrial Robot: An International Journal, vol. 40, no. 1, pp. 59-66, 2013. http://www.emeraldinsight.com/journals.htm? articleid=17072872, last accessed 2021/05/07

[17] Dorhout R\&D LLC and Prospero official website, http://dorhoutrd.com/prospero_robot_farmer, last accessed 2021/05/07
[18] Official website of the Hansjörg Wyss Institute for Biologically Inspired Engineering at Harvard University,

https://wyss.harvard.edu/technology/autonom ous-flying-microrobots-robobees, last accessed 2021/05/07

[19] RIPPA and VIIPA, the University of Sydney's agricultural robots, image acquired from the University of Sydney, https://sydney.edu.au, last accessed 2021/05/07

[20] Krastev, G., Ts. Georgiev, A Prototype of Autonomous Mobile Robot. IN: European Science and Technology - Materials of the X International Research and Practice Conference, Vol. II, May 28th-29th, 2015, Munich, Germany, Vela Verlag Waldkraiburg - Munich - Germany, 2015, pp. 310-314, ISBN 978-3-941352-46-9.

[21] Beloev, I., Kinaneva, D., Georgiev, G., Hristov, G., Zahariev, P., Artificial Intelligence-Driven Autonomous Robot for Precision Agriculture, Acta Technologica Agriculturae, vol.24, no.1, 2021, pp.48-54. https://doi.org/10.2478/ata2021-0008

[22] Webpage of Project "Forest Monitoring System for Early Fire Detection and Assessment in the Balkan-Med Area" (SFEDA), http://www.interreg-balkanmed.eu/approvedproject/22/, last accessed 2021/05/07

[23] Webpage of ALTi UAS, https://www.altiuas.com/, last accessed 2021/05/07

[24] Webpage of the Institute of Mechanics at BAS, https://www.imbm.bas.bg, last accessed 2021/05/15

[25] Yotov, Y., Zlatov, N., Dexterous Flight Telerobotic System with flexible connection, Mechanics of Machines, Scientific journal of the National Committee for Theory of Mechanisms and Machines, Technical University of Varna, Varna, 2019, ISDN: ISSN 0861-9727 